\title{
Pattern Dynamics of Nonlocal Delay SI Epidemic Model with the Growth of the Susceptible following Logistic Mode
}

\author{
Zun-Guang Guo, ${ }^{1,2}$ Jing Li $\mathbb{D},{ }^{3}$ Can Li, ${ }^{1}$ Juan Liang $\mathbb{D},{ }^{1}$ and Yiwei Yan ${ }^{1}$ \\ ${ }^{1}$ Department of Science, Taiyuan Institute of Technology, Taiyuan, Shanxi 030008, China \\ ${ }^{2}$ Department of Computer Science and Technology, North University of China, Taiyuan, Shanxi 030051, China \\ ${ }^{3}$ College of Applied Mathematics, Shanxi University of Finance and Economics, Taiyuan, Shanxi 030006, China
}

Correspondence should be addressed to Jing Li; jingli_2016@126.com

Received 2 May 2020; Accepted 17 June 2020; Published 26 July 2020

Guest Editor: Songbai Guo

Copyright (c) 2020 Zun-Guang Guo et al. This is an open access article distributed under the Creative Commons Attribution License, which permits unrestricted use, distribution, and reproduction in any medium, provided the original work is properly cited.

\begin{abstract}
In this paper, we investigate pattern dynamics of a nonlocal delay SI epidemic model with the growth of susceptible population following logistic mode. Applying the linear stability theory, the condition that the model generates Turing instability at the endemic steady state is analyzed; then, the exact Turing domain is found in the parameter space. Additionally, numerical results show that the time delay has key effect on the spatial distribution of the infected, that is, time delay induces the system to generate stripe patterns with different spatial structures and affects the average density of the infected. The numerical simulation is consistent with the theoretical results, which provides a reference for disease prevention and control.
\end{abstract}

\section{Introduction}

Research on infectious disease models can be traced back to the pioneering work of Kermack and Mckendrick [1,2], and the traditional model is usually described by ordinary differential equations as a result of the spatially homogeneous assumption [3-10]. Considering the instantaneous change, researchers have established epidemic models via impulsive differential equations [11-15]. However, besides the change of time, the spatial distribution of populations has an significant effect on diseases transmission. As a matter of fact, spatial distribution of the population is not homogeneous, but depend on the spatial location and the surrounding environment factors, such as the amount of food, the number of natural enemies, and the number of the infected $[16,17]$. Therefore, considering the space in epidemic models is more suitable for the spread process of infectious diseases. Recently, many reaction-diffusion models have been proposed to study their dynamical behaviors and study the corresponding control measures [18-29].

Since they can reach the current position from any other positions in the whole space when humans or animals are moving in space, which is more reflective for the actual situation, therefore, the nonlocal delay of infectious disease has become a research focus in epidemiology. Wang and $\mathrm{Wu}$ [30] studied the Kermack-Mckendrick SIR model with nonlocal delay and discussed the dynamic properties of disease spatial diffusion. Pan [31] studied the existence of wave front solutions for a class of infectious disease models with nonlocal diffusion and time delay by constructing upper and lower solutions. Zhen et al. [32] also obtained the traveling wave solutions for a class of SIR epidemic models with spatiotemporal delays. Using the upper and lower solutions and its related monotone iterative techniques, Tang et al. [33] studied the sufficient conditions for the global asymptotic stability of the disease-free equilibrium of the bird system.

Most of the abovementioned studies focus on the traveling wave solutions and global stability of the nonlocal epidemic model. However, few literatures study the pattern formation of infectious disease models based on reactiondiffusion equation with nonlocal delay. Pattern dynamics can effectively characterize the spatial distribution of the infected, so as to provide decision-making guidance for the 
government. In fact, in the natural environments with limited resources, the assumption of logistic growth is more consistent with the actual situation [34-37]; then, we assume the susceptible possess the logistic growth in the absence of the infected. In addition, we take the standard incidence ratio to describe the infection between the susceptible and the infected. To this end, we propose the following model:

$$
\left\{\begin{array}{l}
\frac{\partial S}{\partial t}=D_{S} \Delta S+r S\left(\left(1-\frac{S}{K}\right)-\beta S \frac{\int_{-\infty}^{t} \int_{\Omega} Q(x-y, t-s) I(y, s) \mathrm{d} y \mathrm{~d} s}{S+I}-d S-A S\right. \\
\frac{\partial I}{\partial t}=D_{I} \Delta I+\beta S \frac{\int_{-\infty}^{t} \int_{\Omega} Q(x-y, t-s) I(y, s) \mathrm{d} y \mathrm{~d} s}{S+I}-\mu I-d I
\end{array}\right.
$$

with initial boundary value condition

$$
(\vec{n} \cdot \nabla)\left(\begin{array}{l}
S \\
I
\end{array}\right)=0, S(x, 0), I(x, 0) \text { are given, }
$$

and the descriptions of the symbols in model (1) are shown in Table 1. The kernel function $Q(x, t)=(1 / 4 \pi t) \exp \left(-|x|^{2} /\right.$ $4 t)(1 / \tau) \exp (-(t / \tau))$ represents the weight from the other possible positions to the position (HTML translation failed) before time $t$. The nonlocal item.depicts the cumulative number of the infected reaching $x$ position at $t$ time who starts from any $y$ position in the entire space at $s$ time.

$$
\int_{-\infty}^{t} \int_{R^{2}} Q(x-y, t-s) I(y, s) \mathrm{d} s \mathrm{~d} y .
$$

In order to study the spatiotemporal dynamics of model (1), in Section 2, we first obtain the steady states of model (1), linearize the model at the endemic steady state, and then analyze the conditions of system generating Turing instability and parameter space of Turing pattern generated. In Section 3, different stripe patterns are shown for different time delays, which indicates that time delay affects the structures of Turing patterns. In Section 4, the summary and discussion are given.

\section{Linear Analysis and Turing Patterns}

In this section, we define a new variable $V(x, t)$ to replace the nonlocal term $\int_{-\infty}^{t} \int_{R^{2}} \mathrm{Q}(x-y, t-s) I(y, s) \mathrm{d} y \mathrm{~d} s$; then, system (1) can be transformed into 3-variable reactiondiffusion system:

$$
\frac{\partial \mathbf{c}}{\partial t}=\mathbf{F}(\mathbf{c})+D \Delta \mathbf{c}
$$

where

$$
\mathbf{c}=\left(\begin{array}{c}
S \\
I \\
V
\end{array}\right) \text {, }
$$

$$
\begin{aligned}
& \mathbf{F}(\mathbf{c})=\left(\begin{array}{c}
r S\left(1-\frac{S}{K}\right)-\frac{\beta S V}{S+I}-(d+A) S \\
\frac{\beta S V}{S+I}-(\mu+d) I \\
\frac{1}{\tau}(I-V)
\end{array}\right), \\
& D=\left(\begin{array}{ccc}
D_{S} & & \\
& D_{I} & \\
& & 1
\end{array}\right) \text {. }
\end{aligned}
$$

By studying system (4) without diffusion, so the ordinary differential equation is given:

$$
\begin{aligned}
\frac{\mathrm{d} S(t)}{\mathrm{d} t} & =r S(t)\left(1-\frac{S(t)}{K}\right)-\frac{\beta S(t) V(t)}{S(t)+I(t)}-(d+A) S(t), \\
\frac{\mathrm{d} I(t)}{\mathrm{d} t} & =\frac{\beta S(t) V(t)}{S(t)+I(t)}-(\mu+d) I(t), \\
\frac{\mathrm{d} V(t)}{\mathrm{d} t} & =\frac{1}{\tau}(I(t)-V(t)),
\end{aligned}
$$

system (6) has two constant steady states $E_{0}=\left(S_{0}, I_{0}, V_{0}\right)=$ $(K(r-A-d) / r, 0,0)$ and $E_{1}^{*}=\left(S_{1}^{*}, I_{1}^{*}, V_{1}^{*}\right)$, where

$$
\begin{aligned}
S_{1}^{*} & =\frac{K(r+\mu-A-\beta)}{r}, \\
I_{1}^{*} & =\frac{K(r+\mu-A-\beta)(\beta-d-\mu)}{r(d+\mu)}, \\
V_{1}^{*} & =\frac{K(r+\mu-A-\beta)(\beta-d-\mu)}{r(d+\mu)} .
\end{aligned}
$$

According to the actually biological situation, the population number should be nonnegative. So, if $r>A+d$ holds, then $S_{0}>0$, that is, $E_{0}$ is disease-free steady states. In addition, if the condition

$$
\text { (H1) } \quad r>A+\beta-\mu, \quad \beta>\mu+d,
$$


TABle 1: Symbol descriptions of model (1).

\begin{tabular}{lc}
\hline Symbol & Interpretations \\
\hline$S(x, t)$ & The density of the susceptible at position $x\left(x \in R^{2}\right)$ at \\
$I(x, t)$ & The density of the infected at position $x\left(x \in R^{2}\right)$ at time $t$ \\
$A$ & The emigration rate of the susceptible \\
$\Omega$ & The diffusion domain \\
$\vec{n}$ & Unit outward normal to $\partial \Omega$ \\
$\partial \Omega$ & The closed boundary of the diffusion domain $\Omega$ \\
$\Delta$ & Laplace operator $\left(\partial^{2} / \partial X^{2}\right)+\left(\partial^{2} / \partial Y^{2}\right)$ \\
$D_{S}$ & The diffusion rate of the susceptible \\
$D_{I}$ & The diffusion rate of the infected \\
$\beta$ & The infection rate \\
$\mu$ & The mortality rate due to infectious disease caused \\
$d$ & The natural mortality \\
$r$ & The intrinsic growth rate \\
$K$ & The carrying capacity \\
\hline
\end{tabular}

is satisfied, then $S_{1}^{*}>0, I_{1}^{*}>0$, and $V_{1}^{*}>0$, which shows that $E_{1}^{*}$ is the endemic steady state.

The main focus of infectious diseases is to study the outbreak and prevalence, so as to provide theoretical guidance for the prevention and control of infectious diseases. Then, the following contents only study endemic steady state $E_{1}^{*}$.

Through setting

$$
\mathbf{w}=\left(\begin{array}{c}
S-S_{1}^{*} \\
I-I_{1}^{*} \\
V-V_{1}^{*}
\end{array}\right),
$$

we substitute (9) into (4), expand $\mathbf{F}(\mathbf{c})$ at the endemic steady state $E_{1}^{*}$ based on Talyor expansion, and then obtain the following linear equation:

$$
\frac{\partial \mathbf{w}}{\partial t}=D \Delta \mathbf{w}+\mathbf{J w}, \mathbf{J}=\left(\begin{array}{lll}
a_{11} & a_{12} & a_{13} \\
a_{21} & a_{22} & a_{23} \\
a_{31} & a_{32} & a_{33}
\end{array}\right),
$$

where

$$
\begin{aligned}
& a_{11}=\frac{A \beta+\beta^{2}+\beta d-\beta r-d^{2}-2 d \mu-\mu^{2}}{\beta}, \\
& a_{12}=\frac{(\beta-d-\mu)(d+\mu)}{\beta}, \\
& a_{13}=-d-\mu, \\
& a_{21}=\frac{(\beta-d-\mu)^{2}}{\beta}, \\
& a_{22}=-\frac{2 \beta d+2 \beta \mu-d^{2}-2 d \mu-\mu^{2}}{\beta}, \\
& a_{23}=d+\mu, \\
& a_{31}=0 \\
& a_{32}=\frac{1}{\tau} \\
& a_{33}=-\frac{1}{\tau} .
\end{aligned}
$$

In order to solve the solution of system (10) satisfying the boundary condition (2), we first define $\mathbf{W}(x)$ as the timeindependent spatial eigenvalue problem, which satisfies the following system:

$$
\Delta \mathbf{W}+k^{2} \mathbf{W}=0,(\vec{n} \cdot \nabla) \mathbf{W}=0, \quad \text { for } x \text { on } \partial \Omega,
$$

where $k$ is the eigenvalue (i.e., wavenumber).

We now look for solutions $\mathbf{w}(x, t)$ of $(10)$ in the form

$$
\mathbf{w}(x, t)=\left(\begin{array}{c}
C_{1} \\
C_{2} \\
C_{3}
\end{array}\right) e^{\lambda t+i \vec{k} \cdot x},
$$

where $\vec{k}$ is the wave vector with magnitude $k=|\vec{k}|$. By inserting (13) into (10), the characteristic equation matrix is obtained:

$$
|J-\lambda E-D|=0,
$$

which is equivalent as follows:

$$
\lambda^{3}+a_{2}(k) \lambda^{2}+a_{1}(k) \lambda+a_{0}(k)=0,
$$

with

$$
\begin{aligned}
a_{2}(k)= & \left(D_{S}+D_{I}+1\right) k^{2}-A-\beta+d+2 \mu+r+\frac{1}{\tau}, \\
a_{1}(k)= & \left(D_{S}+D_{I}+D_{S} D_{I}\right) k^{4} \\
& -\frac{1}{\beta \tau}\left(\left(A \beta D_{I} \tau+\beta^{2} D_{I} \tau-2 \beta d D_{S} \tau+\beta d D_{I} \tau-2 \beta D_{S} \mu \tau-\beta D_{I} r \tau\right.\right. \\
& +d^{2} D_{S} \tau-d^{2} D_{I} \tau+2 d D_{S} \mu \tau-2 d D_{I} \mu \tau+D_{S} \mu^{2} \tau-D_{I} \mu^{2} \tau+A \beta \tau \\
& \left.\left.+\beta^{2} \tau-\beta d \tau-2 \beta \mu \tau-\beta r \tau-\beta D_{S}-\beta D_{I}\right) k^{2}\right)-\frac{1}{\beta \tau}(2 A \beta d \tau
\end{aligned}
$$




$$
\begin{aligned}
& +2 A \beta \mu \tau-A d^{2} \tau-2 A d \mu \tau-A \mu^{2} \tau+3 \beta^{2} d \tau+3 \beta^{2} \mu \tau-2 \beta d^{2} \tau \\
& -6 \beta d \mu \tau-2 \beta d r \tau-4 \beta \mu^{2} \tau-2 \beta \mu r \tau+d^{2} \mu \tau+d^{2} r \tau+2 d \mu^{2} \tau \\
& \left.+2 d \mu r \tau+\mu^{3} \tau+\mu^{2} r \tau+A \beta+\beta^{2}-\beta \mu-\beta r\right), \\
a_{0}(k)= & D_{S} D_{I} k^{6}-\frac{1}{\beta \tau}\left(\left(A \beta D_{I} \tau+\beta^{2} D_{I} \tau-2 \beta d D_{S} \tau+\beta d D_{I} \tau-2 \beta D_{S} \mu \tau\right.\right. \\
& -\beta D_{I} r \tau+d^{2} D_{S} \tau-d^{2} D_{I} \tau+2 d D_{S} \mu \tau-2 d D_{I} \mu \tau+D_{S} \mu^{2} \tau-D_{I} \mu^{2} \tau \\
& \left.\left.-\beta D_{S} D_{I}\right) k^{4}\right)-\frac{1}{\beta \tau}\left(\left(2 A \beta d \tau+2 A \beta \mu \tau-A d^{2} \tau-2 A d \mu \tau\right.\right. \\
& -A \mu^{2} \tau+3 \beta^{2} d \tau+3 \beta^{2} \mu \tau-2 \beta d^{2} \tau-6 \beta d \mu \tau-2 \beta d r \tau-4 \beta \mu^{2} \tau \\
& -2 \beta \mu r \tau+d^{2} \mu \tau+d^{2} r \tau+2 d \mu^{2} \tau+2 d \mu r \tau+\mu^{3} \tau+\mu^{2} r \tau \\
& +A \beta D_{I}+\beta^{2} D_{I}-\beta d D_{S}+\beta d D_{I}-\beta D_{S} \mu-\beta D_{I} r+d^{2} D_{S} \\
& \left.\left.-d^{2} D_{I}+2 d D_{S} \mu-2 d D_{I} \mu+D_{S} \mu^{2}-D_{I} \mu^{2}\right) k^{2}\right) \\
& -\frac{(d+\mu)(-d+\beta-\mu)(A+\beta-\mu-r)}{\beta \tau},
\end{aligned}
$$

where $a_{2}(k)>0$ for any $k$ because of condition (H1) satisfied.

Moreover, one can obtain the corresponding characteristic equation for system (6) as follows:

$$
\lambda^{3}+a_{2}(0) \lambda^{2}+a_{1}(0) \lambda+a_{0}(0)=0
$$

where

$$
\begin{aligned}
a_{2}(0)= & -A-\beta+d+2 \mu+r+\frac{1}{\tau}, \\
a_{1}(0)= & -\frac{1}{\beta \tau}\left(2 A \beta d \tau+2 d \mu r \tau+\mu^{3} \tau\right. \\
& +\mu^{2} r \tau+A \beta+\beta^{2}-\beta \mu-\beta r \\
& +2 A \beta \mu \tau-A d^{2} \tau-2 A d \mu \tau-A \mu^{2} \tau \\
& +3 \beta^{2} d \tau+3 \beta^{2} \mu \tau-2 \beta d^{2} \tau \\
& -6 \beta d \mu \tau-2 \beta d r \tau-4 \beta \mu^{2} \tau-2 \beta \mu r \tau \\
& \left.+d^{2} \mu \tau+d^{2} r \tau+2 d \mu^{2} \tau\right) \\
& -\frac{(d+\mu)(\beta-d-\mu)(A+\beta-\mu-r)}{\beta \tau} .
\end{aligned}
$$

On the basis of condition (H1), one can derive $a_{2}(0)>0$ and $a_{0}(0)>0$. According to Hurwitz criterion, the condition for the local asymptotic stability of $E_{1}^{*}$ of system (6) is

$$
(\mathrm{H} 2) a_{2}(0) a_{1}(0)-a_{0}(0)>0 \text {. }
$$

Theorem 1. The nonspatial system (6) gives rise to a Hopf bifurcation at endemic steady state $E_{1}^{*}$ if only and if the condition

$$
(H 3) a_{2}(0) a_{1}(0)-a_{0}(0)=0,
$$

is established.

Proof. It is obvious that $a_{2}(0)>0$ and $a_{0}(0)>0$ due to the existence of endemic equilibrium $E_{1}^{*}$ (i.e., (H1)). According to the theorem of [38], when conditions $a_{2}(0)>0, a_{0}(0)>0$, and $a_{2}(0) a_{1}(0)-a_{0}(0)=0$ hold, a Hopf bifurcation occurs for nonspatial system (6).

Next, we find the conditions under which system (4) generates Turing instability near endemic steady state $E_{1}^{*}: E_{1}^{*}$ is locally asymptotic stability for system (6), but $E_{1}^{*}$ loses stability for system (6) with diffusion (i.e., system (4)). Furthermore, we already know $a_{2}(k)>0$ for any $k$, while the signs of $a_{0}(k)$ and $a_{2}(k) a_{1}(k)-a_{0}(k)$ are uncertain. Now, we need to look for the condition that $E_{1}^{*}$ of system (4) becomes unstable. Obviously, Hurwitz criterion is not satisfied, that is, $a_{0}(k)<0$ or $a_{2}(k) a_{1}(k)-a_{0}(k)<0$; then, the corresponding instability conditions are given. Based on such two cases, we derive the following theorems with respect to Turing instability.

Theorem 2. For system (4), if condition (H1) holds, and one of the following conditions

$$
\begin{aligned}
& \text { (C1) } h_{1}<0 \text { and } H_{2}\left(z_{1}\right)<0 \\
& \text { (C2) } h_{1}>0, h_{2}<0,\left(h_{2}\right)^{2}-3 h_{3} h_{1}>0 \text { and } H_{2}\left(z_{1}\right)<0
\end{aligned}
$$

is satisfied, then endemic steady state $E_{1}^{*}$ is unstable for some $k$, where 


$$
z_{1}=\frac{-h_{2}+\sqrt{\left(h_{2}\right)^{2}-3 h_{3} h_{1}}}{3 h_{3}}
$$

Proof. Let $z=k^{2}>0$ and $H_{2}\left(k^{2}\right)=a_{0}(k)$, then $H_{2}(z)=$ $h_{3} z^{3}+h_{2} z^{2}+h_{1} z+h_{0}$, where

$$
\begin{aligned}
& h_{3}=D_{S} D_{I}>0 \text {, } \\
& h_{2}=-\frac{1}{\beta \tau}\left(A \beta D_{I} \tau+\beta^{2} D_{I} \tau-2 \beta d D_{S} \tau+\beta d D_{I} \tau\right. \\
& -2 \beta D_{S} \mu \tau-\beta D_{S} D_{I} \\
& -\beta D_{I} r \tau+d^{2} D_{S} \tau-d^{2} D_{I} \tau+2 d D_{S} \mu \tau \\
& \left.-2 d D_{I} \mu \tau+D_{S} \mu^{2} \tau-D_{I} \mu^{2} \tau\right) \\
& h_{1}=-\frac{1}{\beta \tau}\left(2 A \beta d \tau+2 A \beta \mu \tau-A d^{2} \tau-2 A d \mu \tau\right. \\
& -A \mu^{2} \tau+3 \beta^{2} d \tau+3 \beta^{2} \mu \tau-2 \beta d^{2} \tau-6 \beta d \mu \tau \\
& -2 \beta d r \tau-4 \beta \mu^{2} \tau \\
& -2 \beta \mu r \tau+d^{2} \mu \tau+d^{2} r \tau+2 d \mu^{2} \tau+2 d \mu r \tau+\mu^{3} \tau+\mu^{2} r \tau \\
& +A \beta D_{I}+\beta^{2} D_{I}-\beta d D_{S}+\beta d D_{I}-\beta D_{S} \mu-\beta D_{I} r+d^{2} D_{S} \\
& \left.-d^{2} D_{I}+2 d D_{S} \mu-2 d D_{I} \mu+D_{S} \mu^{2}-D_{I} \mu^{2}\right), \\
& h_{0}=-\frac{(d+\mu)(-d+\beta-\mu)(A+\beta-\mu-r)}{\beta \tau}>0 .
\end{aligned}
$$

Considering the properties of cubic polynomials, the coefficient of the third order of $\mathrm{H}_{2}(z)$ is greater than zero and $H_{2}(0)=h_{0}>0$. By solving the first-order derivative $H_{2}(z)$ about $z$, namely, $H_{2}^{\prime}(z)=3 h_{3} z^{2}+2 h_{2} z+h_{1}=0$, we can deduce

$$
z_{2}=\frac{-h_{2}-\sqrt{\left(h_{2}\right)^{2}-3 h_{3} h_{1}}}{3 h_{3}} \leq z_{1}=\frac{-h_{2}+\sqrt{\left(h_{2}\right)^{2}-3 h_{3} h_{1}}}{3 h_{3}} .
$$

(i) If condition $h_{1}<0$ holds, then one can obtain $z_{2}<0<z_{1}$, which further shows that $z_{1}$ is the minimum point. Then, we can get $a_{2}(k)<0$ for some $k$ when $H_{2}\left(z_{2, \min }\right)<0$ combining with $H_{2}(0)=h_{0}>0$ (see Figure 1(a)).

(ii) If conditions $h_{1}>0, h_{2}<0$, and $\left(h_{2}\right)^{2}-3 h_{3} h_{1}>0$ hold, then we can get $0<z_{2}<z_{1}$, and $z_{2}$ and $z_{1}$ are maximum and minimum points, respectively. Furthermore, according to $H_{2}\left(z_{1}\right)<0$, one can derive that $a_{2}(k)<0$ for some $k$ (see Figure $1(\mathrm{~b})$ ).

Obviously, Hurwitz criterion does not hold for Case (i) or Case (ii), and then the endemic steady state $E_{1}^{*}$ is unstable for system (4).

Thus, the conditions of system (4) generating Turing instability is given:

$\begin{cases}(\mathrm{H} 1), & (\mathrm{H} 2), \\ (\mathrm{C} 1) \text { or, } & (\mathrm{C} 2) .\end{cases}$

In addition, we set $H_{3}(z)=a_{2}(k) a_{1}(k)-a_{0}(k)$ and $z=k^{2}>0$, that is,

$$
H_{3}(z)=h_{q_{3}} z^{3}+h_{q_{2}} z^{2}+h_{q_{1}} z+h_{q_{0}},
$$

where

$$
\begin{aligned}
h_{q_{3}}= & \left(D_{I}+1\right)\left(D_{S}+1\right)\left(D_{S}+D_{I}\right)>0, \\
h_{q_{2}}= & -\frac{1}{\tau \beta}\left(2 A \beta D_{S} D_{I} \tau+A \beta D_{I}^{2} \tau+2 \beta^{2} D_{S} D_{I} \tau+\beta^{2} D_{I}^{2} \tau-2 \beta d D_{S}^{2} \tau\right. \\
& -2 \beta d D_{S} D_{I} \tau+\beta d D_{I}^{2} \tau-2 \beta D_{S}^{2} \mu \tau-4 \beta D_{S} D_{I} \mu \tau-2 \beta D_{S} D_{I} r \tau \\
& -\beta D_{I}^{2} r \tau+d^{2} D_{S}^{2} \tau-d^{2} D_{I}^{2} \tau+2 d D_{S}^{2} \mu \tau-2 d D_{I}^{2} \mu \tau+D_{S}^{2} \mu^{2} \tau \\
& -D_{I}^{2} \mu^{2} \tau+2 A \beta D_{S} \tau+2 A \beta D_{I} \tau+2 \beta^{2} D_{S} \tau+2 \beta^{2} D_{I} \tau-2 \beta d D_{S} \tau \\
& -2 \beta d D_{I} \tau-4 \beta D_{S} \mu \tau-2 \beta D_{S} r \tau-4 \beta D_{I} \mu \tau-2 \beta D_{I} r \tau+A \beta \tau \\
& \left.+\beta^{2} \tau-\beta d \tau-\beta D_{S}^{2}-2 \beta D_{S} D_{I}-\beta D_{I}^{2}-2 \beta \mu \tau-\beta r \tau-2 \beta D_{S}-2 \beta D_{I}\right), \\
& 1 \\
h_{q_{1}} & \frac{1}{\tau^{2} \beta}\left(A^{2} \beta D_{I} \tau^{2}+2 A \beta^{2} D_{I} \tau^{2}-4 A \beta d D_{S} \tau^{2}-2 A \beta d D_{I} \tau^{2}-4 A \beta D_{S} \mu \tau^{2}\right. \\
& -4 A \beta D_{I} \mu \tau^{2}-2 A \beta D_{I} r \tau^{2}+2 A d^{2} D_{S} \tau^{2}+4 A d D_{S} \mu \tau^{2}+2 A D_{S} \mu^{2} \tau^{2} \\
& +\beta^{3} D_{I} \tau^{2}-5 \beta^{2} d D_{S} \tau^{2}-3 \beta^{2} d D_{I} \tau^{2}-5 \beta^{2} D_{S} \mu \tau^{2}-5 \beta^{2} D_{I} \mu \tau^{2} \\
& -2 \beta^{2} D_{I} r \tau^{2}+5 \beta d^{2} D_{S} \tau^{2}+14 \beta d D_{S} \mu \tau^{2}+4 \beta d D_{S} r \tau^{2}+2 \beta d D_{I} \mu \tau^{2}
\end{aligned}
$$




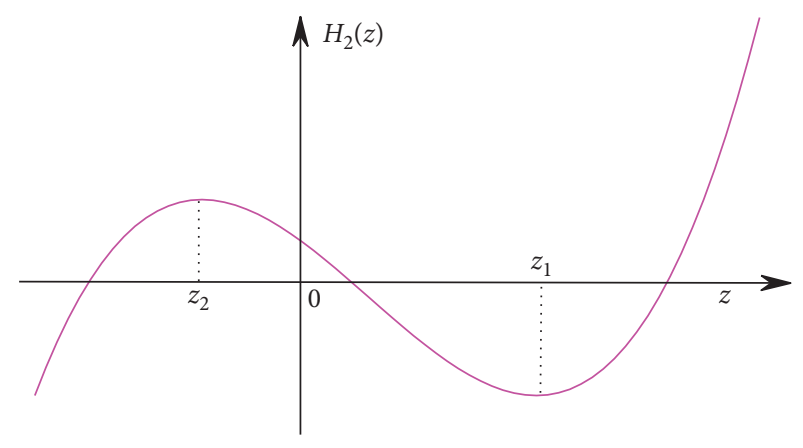

(a)

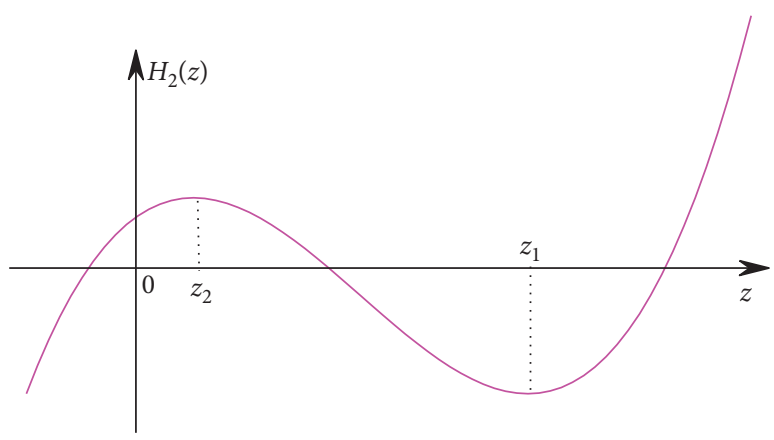

(b)

Figure 1: The diagrams of the cubic function $H_{2}(z)$ in Theorem 1: (a) case (i) and (b) case (ii).

$$
\begin{aligned}
& +2 \beta d D_{I} r \tau^{2}+9 \beta D_{S} \mu^{2} \tau^{2}+4 \beta D_{S} \mu r \tau^{2}+3 \beta D_{I} \mu^{2} \tau^{2}+4 \beta D_{I} \mu r \tau^{2} \\
& +\beta D_{I} r^{2} \tau^{2}-d^{3} D_{S} \tau^{2}+d^{3} D_{I} \tau^{2}-5 d^{2} D_{S} \mu \tau^{2}-2 d^{2} D_{S} r \tau^{2} \\
& +3 d^{2} D_{I} \mu \tau^{2}-7 d D_{S} \mu^{2} \tau^{2}-4 d D_{S} \mu r \tau^{2}+3 d D_{I} \mu^{2} \tau^{2}-3 D_{S} \mu^{3} \tau^{2} \\
& -2 D_{S} \mu^{2} r \tau^{2}+D_{I} \mu^{3} \tau^{2}+A^{2} \beta \tau^{2}+2 A \beta^{2} \tau^{2}-2 A \beta d \tau^{2}-4 A \beta \mu \tau^{2} \\
& -2 A \beta r \tau^{2}+\beta^{3} \tau^{2}-2 \beta^{2} d \tau^{2}-4 \beta^{2} \mu \tau^{2}-2 \beta^{2} r \tau^{2}+\beta d^{2} \tau^{2} \\
& +4 \beta d \mu \tau^{2}+2 \beta d r \tau^{2}+4 \beta \mu^{2} \tau^{2}+4 \beta \mu r \tau^{2}+\beta r^{2} \tau^{2}-2 A \beta D_{S} \tau \\
& -2 A \beta D_{I} \tau-2 \beta^{2} D_{S} \tau-2 \beta^{2} D_{I} \tau+2 \beta d D_{S} \tau+\beta d D_{I} \tau+4 \beta D_{S} \mu \tau \\
& +2 \beta D_{S} r \tau+3 \beta D_{I} \mu \tau+2 \beta D_{I} r \tau-2 A \beta \tau-2 \beta^{2} \tau+\beta d \tau \\
& \left.+3 \beta \mu \tau+2 \beta r \tau+\beta D_{S}+\beta D_{I}\right), \\
& h_{q_{0}}=\frac{1}{\tau^{2} \beta}\left(2 A^{2} \beta d \tau^{2}+2 A^{2} \beta \mu \tau^{2}-A^{2} d^{2} \tau^{2}-2 A^{2} d \mu \tau^{2}-A^{2} \mu^{2} \tau^{2}\right. \\
& +5 A \beta^{2} d \tau^{2}+5 A \beta^{2} \mu \tau^{2}-5 A \beta d^{2} \tau^{2}-14 A \beta d \mu \tau^{2}-4 A \beta d r \tau^{2} \\
& -9 A \beta \mu^{2} \tau^{2}-4 A \beta \mu r \tau^{2}+A d^{3} \tau^{2}+5 A d^{2} \mu \tau^{2}+2 A d^{2} r \tau^{2} \\
& +7 A d \mu^{2} \tau^{2}+4 A d \mu r \tau^{2}+3 A \mu^{3} \tau^{2}+2 A \mu^{2} r \tau^{2}+3 \beta^{3} d \tau^{2} \\
& +3 \beta^{3} \mu \tau^{2}-5 \beta^{2} d^{2} \tau^{2}-15 \beta^{2} d \mu \tau^{2}-5 \beta^{2} d r \tau^{2}-10 \beta^{2} \mu^{2} \tau^{2} \\
& -5 \beta^{2} \mu r \tau^{2}+2 \beta d^{3} \tau^{2}+11 \beta d^{2} \mu \tau^{2}+5 \beta d^{2} r \tau^{2}+18 \beta d \mu^{2} \tau^{2} \\
& +14 \beta d \mu r \tau^{2}+2 \beta d r^{2} \tau^{2}+9 \beta \mu^{3} \tau^{2}+9 \beta \mu^{2} r \tau^{2}+2 \beta \mu r^{2} \tau^{2} \\
& -d^{3} \mu \tau^{2}-d^{3} r \tau^{2}-4 d^{2} \mu^{2} \tau^{2}-5 d^{2} \mu r \tau^{2}-d^{2} r^{2} \tau^{2}-5 d \mu^{3} \tau^{2} \\
& -7 d \mu^{2} r \tau^{2}-2 d \mu r^{2} \tau^{2}-2 \mu^{4} \tau^{2}-3 \mu^{3} r \tau^{2}-\mu^{2} r^{2} \tau^{2}+A^{2} \beta \tau \\
& +2 A \beta^{2} \tau-2 A \beta d \tau-4 A \beta \mu \tau-2 A \beta r \tau+\beta^{3} \tau-3 \beta^{2} d \tau \\
& -5 \beta^{2} \mu \tau-2 \beta^{2} r \tau+\beta d^{2} \tau+4 \beta d \mu \tau+2 \beta d r \tau+4 \beta \mu^{2} \tau \\
& \left.+4 \beta \mu r \tau+\beta r^{2} \tau-A \beta-\beta^{2}+\beta \mu+\beta r\right) \text {. }
\end{aligned}
$$


Through some analyses, we can give the following conclusion.

Theorem 3. For system (4), suppose that the following holds

(C3) $h_{q_{1}}<0, h_{q_{0}}>0$, and $H_{3}\left(z_{3}\right)<0$

(C4) $h_{q_{1}}>0, h_{q_{0}}>0, h_{q_{2}}<0,\left(h_{q_{2}}\right)^{2}-3 h_{q_{3}} h_{q_{1}}>0$, and $H_{3}\left(z_{3}\right)<0$

Then, endemic steady state $E_{1}^{*}$ becomes unstable for some $k$, where

$$
z_{3}=\frac{-h_{q_{2}}+\sqrt{\left(h_{q_{2}}\right)^{2}-3 h_{q_{3}} h_{q_{1}}}}{3 h_{q_{3}}}
$$

Given that the cubic polynomial functions $H_{2}(z)$ and $H_{3}(z)$ have similar structures and conditions, thus the proof process of Theorem 3 is similar to that of Theorem 2; then, one can easily obtain the above result.

Therefore, we derive another condition for system (4) to give rise to Turing instability:

$$
\begin{cases}(\mathrm{H} 1), & (\mathrm{H} 2), \\ (\mathrm{C} 3) \text { or, } & (\mathrm{C} 4) .\end{cases}
$$

\section{Numerical Results}

In this section, we will carry out numerical simulations for system (4) with Neumann boundary conditions, which will be used to illustrate our theoretical analysis. For the convenience of numerical simulation, we select two-dimensional space area $\Omega=[0, a] \times[0, a]$ and set the same space step $h$ in $X$ and $Y$ directions of the space area. Simultaneously, time step is fixed as $\Delta t$. Then, the mesh generation is given:

$$
\begin{aligned}
& x_{i}=i h, \quad i=0,1,2, \ldots, J, \\
& y_{j}=j h, \quad j=0,1,2, \ldots, J, J h=a, \\
& t_{n}=n \Delta t, \quad n=0,1,2, \ldots .
\end{aligned}
$$

$$
\mathbf{F}(\mathbf{c}) \text { in system (4) is relabeled as } \mathbf{F}(\mathbf{c})=\left(\begin{array}{l}
f_{1}(S, I, V) \\
f_{2}(S, I, V) \\
f_{3}(S, I, V)
\end{array}\right),
$$
and the following finite difference scheme is adopted for system (4):

$$
\left\{\begin{array}{l}
\frac{S_{i, j}^{n+1}-S_{i, j}^{n}}{\Delta t}=D_{S}\left(\frac{S_{i+1, j}^{n}-2 S_{i, j}^{n}+S_{i-1, j}^{n}}{h^{2}}+\frac{S_{i, j+1}^{n}-2 S_{i, j}^{n}+S_{i, j-1}^{n}}{h^{2}}\right)+f_{1}\left(S_{i, j}^{n}, I_{i, j}^{n}, V_{i, j}^{n}\right), \\
\frac{I_{i, j}^{n+1}-I_{i, j}^{n}}{\Delta t}=D_{I}\left(\frac{I_{i+1, j}^{n}-2 I_{i, j}^{n}+I_{i-1, j}^{n}}{h^{2}}+\frac{I_{i, j+1}^{n}-2 I_{i, j}^{n}+I_{i, j-1}^{n}}{h^{2}}\right)+f_{2}\left(S_{i, j}^{n}, I_{i, j}^{n}, V_{i, j}^{n}\right), \\
\frac{V_{i, j}^{n+1}-V_{i, j}^{n}}{\Delta t}=\frac{V_{i+1, j}^{n}-2 V_{i, j}^{n}+V_{i-1, j}^{n}}{h^{2}}+\frac{V_{i, j+1}^{n}-2 V_{i, j}^{n}+V_{i, j-1}^{n}}{h^{2}}+f_{3}\left(S_{i, j}^{n}, I_{i, j}^{n}, V_{i, j}^{n}\right),
\end{array}\right.
$$

where $S_{i, j}^{n}, I_{i, j}^{n}$, and $V_{i, j}^{n}$ represent the approximate value of $S\left(x_{i}, y_{j}, t_{n}\right), I\left(x_{i}, y_{j}, t_{n}\right)$, and $V\left(x_{i}, y_{j}, t_{n}\right)$, respectively. In the simulation process, the initial value is a small random disturbance at endemic steady state $E_{1}^{*}$, and the area range of individuals' activity is $\Omega=[0,100] \times[0,100]$. The space region is evenly divided into $100 \times 100$ grids, and the corresponding space step is $h=1$. The time interval is $[0,5000]$, and the time step is set to $\Delta t=0.005$. The first-order difference is used for the first-order derivative of the time, and the second-order derivative of the space is discretized by the center difference method in process of numerical simulation. For infectious diseases, the spatial distribution of infected individuals is a key issue for the control of infectious disease. Turing pattern can visually describe the spatial distribution of infected individuals. The focus of our simulation is how nonlocal delay affects spatial distribution of infected individuals. We thus take parameter value as $K=1000, d=0.3$, $\mu=0.2, r=0.8, D_{S}=1, D_{I}=10, A=0.4$, and $\beta=0.58$; time delay $\tau$ is selected as the control parameter. Figures 2 and 3 are the time sequence diagram of spatial distribution of the density of the infected for time delays $\tau=0.1$ and $\tau=0.6$, respectively. It can be seen from these two figures that spatial distribution of the density of the infected evolves with time, which finally presents a regular nonuniform macroscopic structure in limited time, namely, stripe patterns, which indicates the number of infected individuals gradually increases over time and eventually gathers together. Under different time delay parameters, the infected finally forms different spatial distribution structures in the first row of Figure 4, which are called strip patterns. These patterns are further presented in three-dimensional space in the second row of Figure 4, which directly reflects the change of the density of the infected with space. Figure 5 shows the relationship between the average density of the infected and time delay $\tau$, that is, the average density of the infected decreases with the increase of time delay $\tau$ ( $\tau$ is less than the 


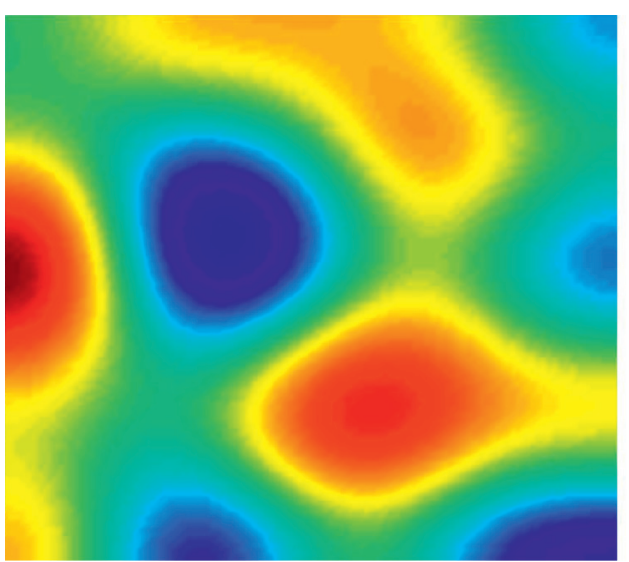

(a)

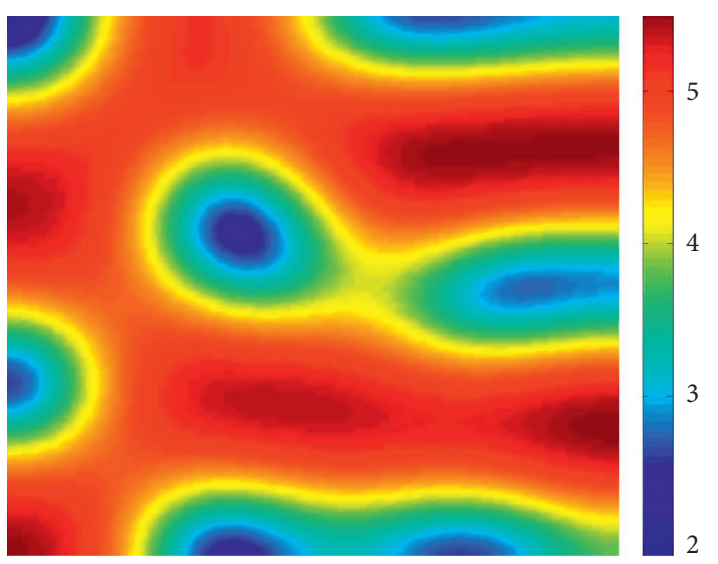

(c)

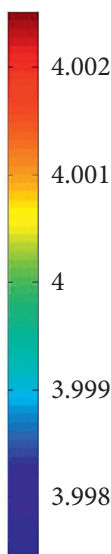

3.998
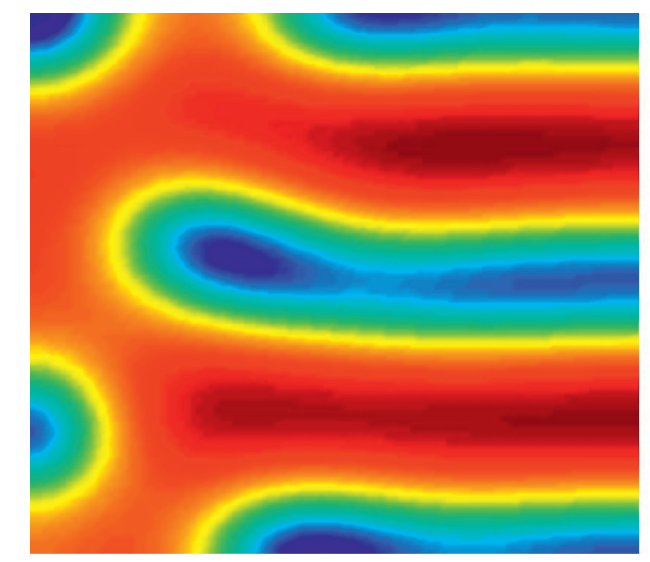

(d)

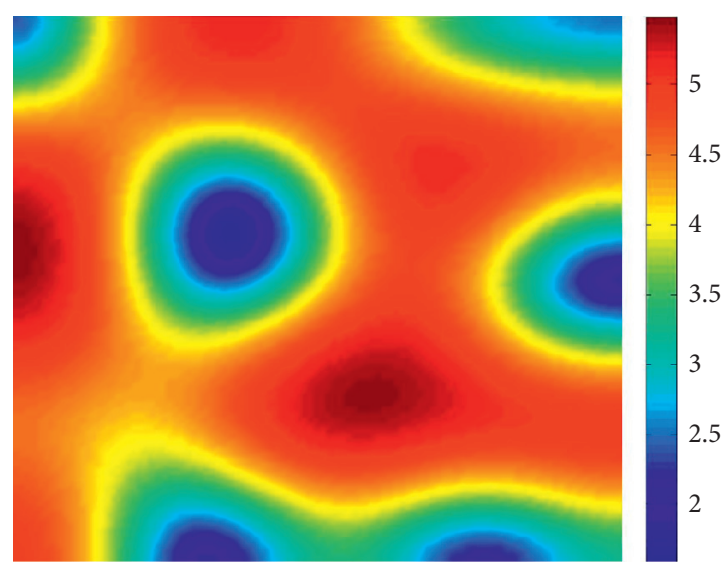

(b)

Figure 2: The spatial evolution of the density of the infected with time for time delay $\tau=0.1$ : (a) $t=90000$; (b) $t=200000$; (c) $t=300000$; (d) $t=1000000$.

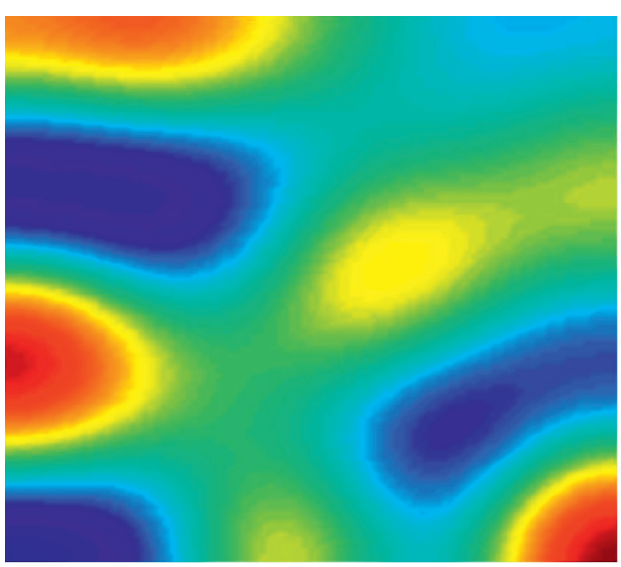

(a)

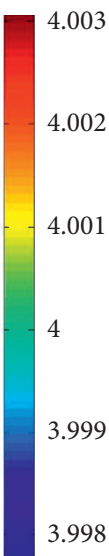

3.998

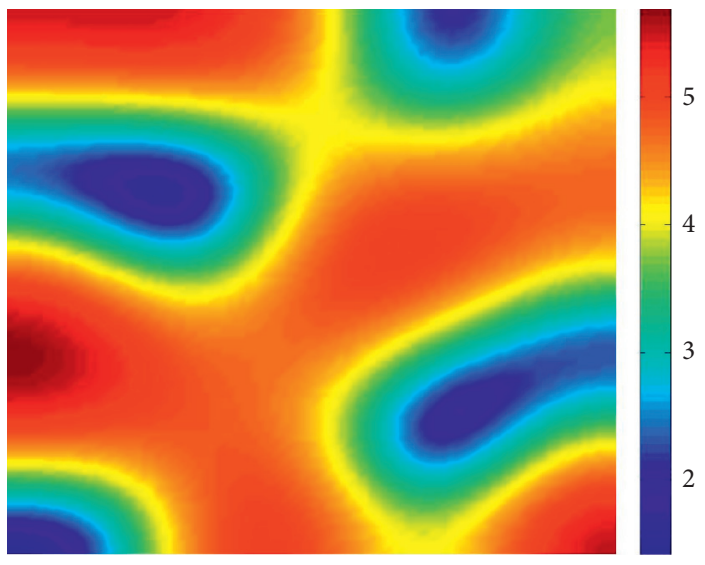

(b)

FIgure 3: Continued. 


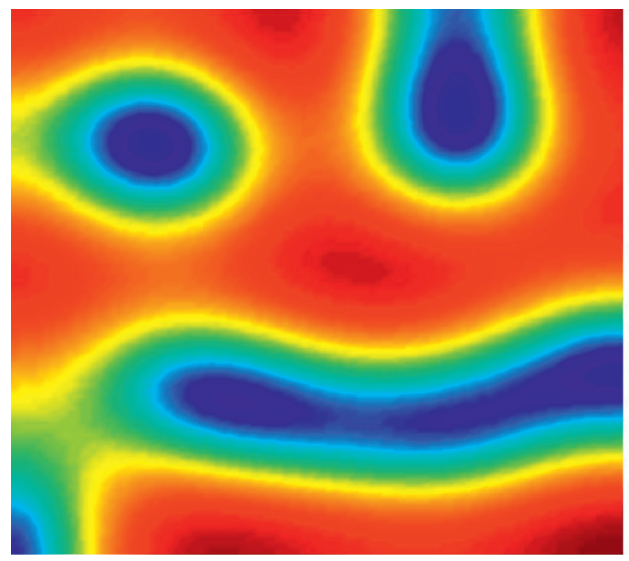

(c)
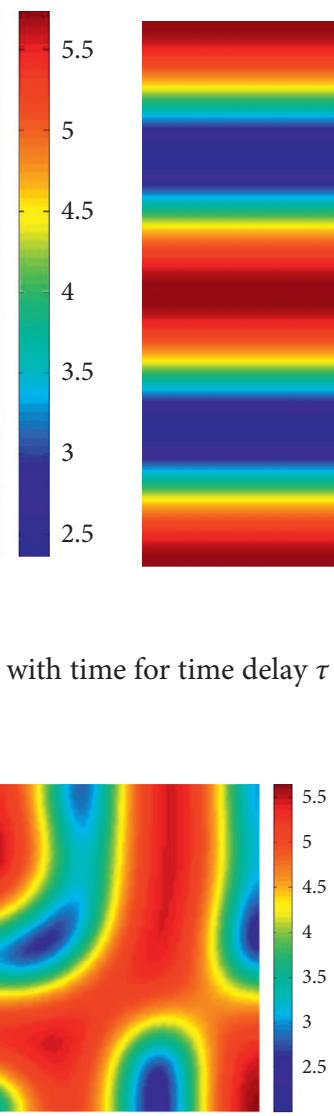

(b)

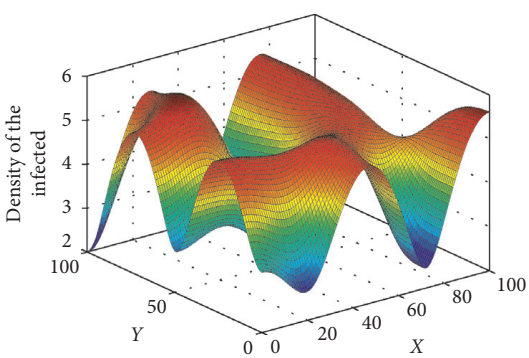

(e)

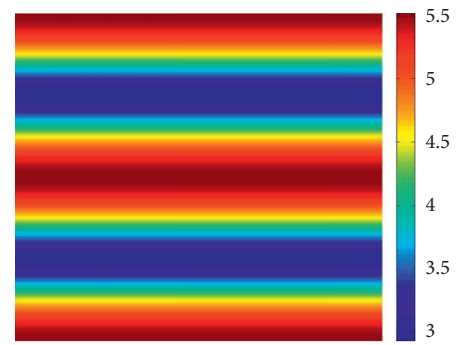

(c)

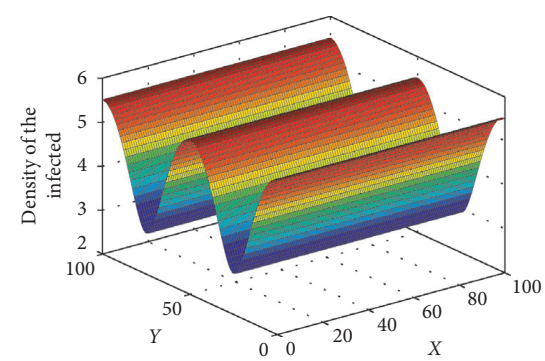

(f)

FIGURE 4: The first row shows stripe patterns with three different spatial structures in two-dimensional space for different time delay parameters: (a and d) $\tau=0.1$; (b and e) $\tau=0.2$; (c and f) $\tau=0.3$. These patterns are further presented in a three-dimensional space in the second row.

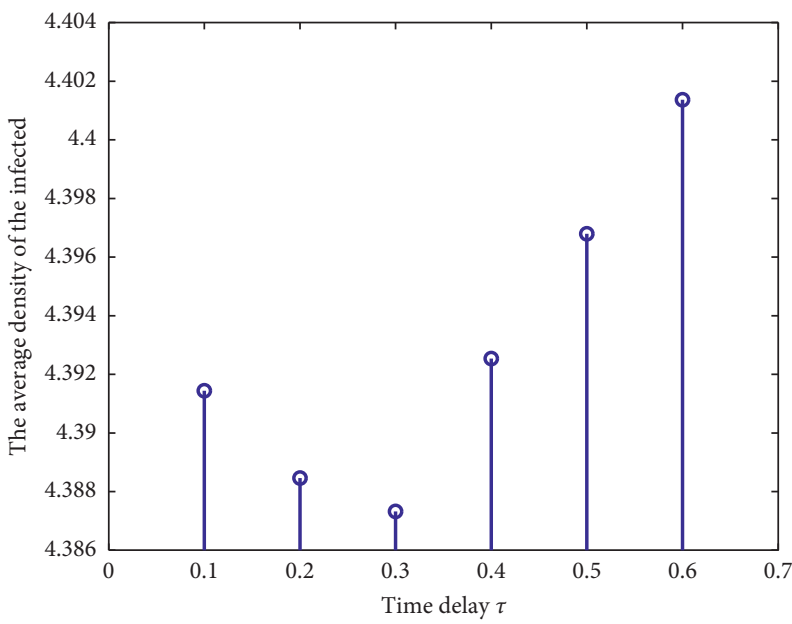

FIGURE 5: The relationship between the average density of the infected and time delay $\tau$. 
critical value), while the opposite case appears when $\tau$ is greater than the critical value.

\section{Conclusions and Discussion}

This paper investigates spatiotemporal dynamics of the SI epidemic model with nonlocal effect and the growth of the susceptible population following logistic mode, and we obtain two spatially homogeneous steady states including disease-free steady state and endemic steady state. We mainly analyze the dynamical behavior near endemic steady state-Turing instability. In general, the condition of system generating Turing instability is that the endemic steady state is stable in the system without diffusion (i.e., ordinary equation system), but becomes instability in the system with diffusion (i.e., reaction-diffusion system); then, the system finally gives rise to stable spatial structure, which is called Turing pattern. The conditions of system (4) generating Turing instability are (24) or (28) based on Hurwitz criterion, and the parameter space of the existence of Turing pattern is given by condition (24). While the corresponding parameter space is difficult to obtain because of the complexity condition (28), which will be carried out in the following work. Time delay widely exists in the process of disease transmission, such as incubation period and immune period. We thus study the influence of time delay on infectious diseases in numerical simulations, find that the average density of the infected firstly decreases, and then increase with the increase of time delay, which provides a theoretical support for disease control. At the same time, we simulate the evolution of spatial distribution of the density of the infected under different time delays, and the spatial distribution of the density of the infected finally forms stripe pattern with different spatial structure, which does not change with time. The high incidence area of infectious diseases is found, which also provides data information for prevention and control of infectious disease.

In the research process of pattern dynamics of system (4), we consider a special form of kernel function so that the nonlocal delay system could be transformed into general reaction-diffusion system; then, pattern dynamics can be followed. In fact, there are some other forms of kernel functions in the biological system, but different forms shall induce nonlocal delay system to convert into different differential system; thus, this is a very meaningful work to study the effects of different kernel functions on the pattern dynamics in the future.

\section{Data Availability}

No data were used to support this study.

\section{Conflicts of Interest}

The authors declare that they have no conflicts of interest.

\section{Acknowledgments}

The project was funded by the Natural Science Foundation of Shanxi Province (Grant nos. 201901D111322 and
201901D211411), Universities' Science and Technology Innovation Item of Shanxi Province (Grant no. 2019L0472), Program for the (Reserved) Discipline Leaders of Taiyuan Institute of Technology (Grant no. 2018008), Natural Science Foundation of Taiyuan Institute of Technology (Grant no. 2016LZ02), and Graduate Students' Education Innovation Item of Shanxi Province (Grant no. 2016BY120).

\section{References}

[1] W. O. Kermack and A. G. McKendrick, "A contribution to the mathematical theory of epidemics," Proceedings of the Royal Society of London. Series A, Containing Papers of a Mathematical and Physical Character, vol. 115, no. 772, pp. 700-721, 1927.

[2] W. O. Kermack and A. G. McKendrick, "Contributions to the mathematical theory of epidemics-I," Bulletin of Mathematical Biology, vol. 53, no. 1-2, pp. 33-55, 1991.

[3] L. Li, G.-Q. Sun, and Z. Jin, "Bifurcation and chaos in an epidemic model with nonlinear incidence rates," Applied Mathematics and Computation, vol. 216, no. 4, pp. 1226-1234, 2010.

[4] Y. Kang and C. Castillo-Chavez, "Dynamics of SI models with both horizontal and vertical transmissions as well as Allee effects," Mathematical Biosciences, vol. 248, pp. 97-116, 2014.

[5] G.-Q. Sun and Z.-K. Zhang, "Global stability for a sheep brucellosis model with immigration," Applied Mathematics and Computation, vol. 246, no. 1, pp. 336-345, 2014.

[6] S. Usaini, R. Anguelov, and S. M. Garba, "Dynamics of SI epidemic with a demographic Allee effect," Theoretical Population Biology, vol. 106, pp. 1-13, 2015.

[7] J. P. Tripathi and S. Abbas, "Global dynamics of autonomous and nonautonomous SI epidemic models with nonlinear incidence rate and feedback controls," Nonlinear Dynamics, vol. 86, no. 1, pp. 337-351, 2016.

[8] G.-Q. Sun, J.-H. Xie, S.-H. Huang, Z. Jin, M.-T. Li, and L. Liu, "Transmission dynamics of cholera: mathematical modeling and control strategies," Communications in Nonlinear Science and Numerical Simulation, vol. 45, pp. 235-244, 2017.

[9] L. Li, J. Zhang, C. Liu, H.-T. Zhang, Y. Wang, and Z. Wang, "Analysis of transmission dynamics for Zika virus on networks," Applied Mathematics and Computation, vol. 347, pp. 566-577, 2019.

[10] L. Chen and J. Sun, "Global stability of an SI epidemic model with feedback controls," Applied Mathematics Letters, vol. 28, pp. 53-55, 2014.

[11] J. Jiao, L. Chen, and G. Luo, "An appropriate pest management SI model with biological and chemical control concern," Applied Mathematics and Computation, vol. 196, no. 1, pp. 285-293, 2008.

[12] K. M. Fuhrman, I. G. Lauko, and G. A. Pinter, "Asymptotic behavior of an SI epidemicmodel with pulse removal," Mathematical and Computer Modelling, vol. 40, no. 3-4, pp. 371-386, 2004.

[13] K. Y. Liu, T. Q. Zhang, and L. S. Chen, "State-dependent pulse vaccination and therapeutic strategy in an SI epidemic model with nonlinear incidence rate," Computational and Mathematical Methods in Medicine, vol. 2019, Article ID 3859815, 10 pages, 2019.

[14] B. Liu, Y. Duan, and S. Luan, "Dynamics of an SI epidemic model with external effects in a polluted environment," Nonlinear Analysis: Real World Applications, vol. 13, no. 1, pp. 27-38, 2012. 
[15] Y. Pei, S. Liu, C. Li, and L. Chen, "The dynamics of an impulsive delay SI model with variable coefficients," Applied Mathematical Modelling, vol. 33, no. 6, pp. 2766-2776, 2009.

[16] L. Li, J. Zhen, and S. Gui-Quan, "Spatial pattern of an epidemic model with cross-diffusion," Chinese Physics Letters, vol. 25, no. 9, pp. 3500-3503, 2008.

[17] Y. Song, H. Jiang, H. Jiang, and Y. Yuan, "Turing-hopf bifurcation in the reaction-diffusion system with delay and application to a diffusive predator-prey model," Journal of Applied Analysis \& Computation, vol. 9, no. 3, pp. 1132-1164, 2019.

[18] Y. Wang, J. Wang, and L. Zhang, "Cross diffusion-induced pattern in an SI model," Applied Mathematics and Computation, vol. 217, no. 5, pp. 1965-1970, 2010.

[19] G.-Q. Sun, "Pattern formation of an epidemic model with diffusion," Nonlinear Dynamics, vol. 69, no. 3, pp. 1097-1104, 2012.

[20] X. Tang and Y. Song, "Cross-diffusion induced spatiotemporal patterns in a predator-prey model with herd behavior," Nonlinear Analysis: Real World Applications, vol. 24, pp. 36-49, 2015.

[21] W. Ding, W. Z. Huang, W. Huang, and S. Kansakar, "Traveling wave solutions for a diffusive SIS epidemic model," Discrete \& Continuous Dynamical Systems - B, vol. 18, no. 5, pp. 1291-1304, 2013.

[22] J. Li, G.-Q. Sun, and Z. Jin, "Pattern formation of an epidemic model with time delay," Physica A: Statistical Mechanics and Its Applications, vol. 403, pp. 100-109, 2014.

[23] G. Q. Sun, S. L. Wang, Q. Ren, Z. Jin, and Y. P. Wu, "Effects of time delay and space on herbivore dynamics: linking inducible defenses of plants to herbivore outbreak," Scientific Reports, vol. 5, no. 1, Article ID 11246, 2015.

[24] T. Zhang, W. Wang, and K. Wang, "Minimal wave speed for a class of non-cooperative diffusion-reaction system," Journal of Differential Equations, vol. 260, no. 3, pp. 2763-2791, 2016.

[25] G.-Q. Sun, C.-H. Wang, and Z.-Y. Wu, "Pattern dynamics of a Gierer-Meinhardt model with spatial effects," Nonlinear Dynamics, vol. 88, no. 2, pp. 1385-1396, 2017.

[26] Z. Lin and H. Zhu, "Spatial spreading model and dynamics of West Nile virus in birds and mosquitoes with free boundary," Journal of Mathematical Biology, vol. 75, no. 6-7, pp. 13811409, 2017.

[27] G.-Q. Sun, C.-H. Wang, L.-L. Chang, Y.-P. Wu, L. Li, and Z. Jin, "Effects of feedback regulation on vegetation patterns in semi-arid environments," Applied Mathematical Modelling, vol. 61, pp. 200-215, 2018.

[28] P. Magal, G. F. Webb, and Y. Wu, "On the basic reproduction number of reaction-diffusion epidemic models," SIAM Journal on Applied Mathematics, vol. 79, no. 1, pp. 284-304, 2019.

[29] J. Ge, C. Lei, and Z. Lin, "Reproduction numbers and the expanding fronts for a diffusion-advection SIS model in heterogeneous time-periodic environment," Nonlinear Analysis: Real World Applications, vol. 33, pp. 100-120, 2017.

[30] Z.-C. Wang and J. Wu, "Travelling waves of a diffusive Kermack-McKendrick epidemic model with non-local delayed transmission," Proceedings of the Royal Society A: Mathematical, Physical and Engineering Sciences, vol. 466, no. 2113, pp. 237-261, 2009.

[31] S. X. Pan, "Traveling wave fronts in an epidemic model with nonlocal diffusion and time delay," International Journal of Mathematical Analysis, vol. 2, no. 21-24, pp. 1083-1088, 2008.

[32] Z. Zhen, J. Wei, L. Tian, J. Zhou, and W. Chen, "Wave propagation in a diffusive SIR epidemic model with spatiotemporal delay," Mathematical Methods in the Applied Sciences, vol. 41, no. 16, pp. 7074-7098, 2018.

[33] Q. Tang, J. Ge, and Z. Lin, "An SEI-SI avian-human influenza model with diffusion and nonlocal delay," Applied Mathematics and Computation, vol. 247, pp. 753-761, 2014.

[34] F. M. Hilker, M. Langlais, and H. Malchow, "The allee effect and infectious diseases: extinction, multistability, and the (Dis-) appearance of oscillations," The American Naturalist, vol. 173, no. 1, pp. 72-88, 2009.

[35] L. Cai, G. Chen, and D. Xiao, "Multiparametric bifurcations of an epidemiological model with strong Allee effect," Journal of Mathematical Biology, vol. 67, no. 2, pp. 185-215, 2013.

[36] L. Q. Gao and H. W. Hethcote, "Disease transmission models with density-dependent demographics," Journal of Mathematical Biology, vol. 30, no. 7, pp. 717-731, 1992.

[37] J. Li, Z. Jin, Z. Jin, G.-Q. Sun, and L.-P. Song, "Pattern dynamics of a delayed eco-epidemiological model with disease in the predator," Discrete \& Continuous Dynamical System$s-S$, vol. 10, no. 5, pp. 1025-1042, 2017.

[38] P. Yu, "Closed-form conditions of bifurcation points for general differential equations," International Journal of Bifurcation and Chaos, vol. 15, no. 4, pp. 1467-1483, 2005. 